\title{
Physical therapy is targeted and adjusted over time for the rehabilitation of locomotor function in acute spinal cord injury interventions in physical and sports therapy
}

\author{
Martina Franz ${ }^{1}$ Lea Richner ${ }^{1}$ - Markus Wirz ${ }^{2}$ Anne von Reumont ${ }^{3,4} \cdot$ Ulla Bergner $^{4,5} \cdot$ Tanja Herzog $^{1}$. \\ Werner Popp ${ }^{1} \cdot$ Kathrin Bach $^{3,4} \cdot$ Norbert Weidner $^{3,4} \cdot$ Armin Curt $^{1,4}$
}

Received: 19 April 2017 / Revised: 20 August 2017 / Accepted: 20 August 2017 / Published online: 23 October 2017

(C) International Spinal Cord Society 2018

\begin{abstract}
Study design Prospective observational multicenter study.

Objectives Investigation of content, duration and adjustment of physical therapy for the rehabilitation of ambulation in acute spinal cord injury (SCI).
\end{abstract}

Setting European Multicenter Study of SCI (EMSCI).

Methods Physical therapy interventions during acute in-patient rehabilitation of eighty incomplete SCI patients (AIS B, C, D all lesion levels) were recorded using the SCI - Intervention Classification System. Mobility was documented using the Spinal Cord Independence Measurement (SCIM III), demographics and clinical data were retrieved from the EMSCI database.

Results Overall recovery of locomotor function was categorized into three outcome groups (G1-G3). Of 76 initial wheelchair-using patients, $53.9 \%$ remained wheelchair user (G1), 25\% regained moderate (G2) and $21.1 \%$ good walking (G3) capability. Strength training was the most frequently applied intervention of body function/-structure across all outcome groups (about 30\% of all interventions), while interventions focusing on muscle tone and respiration were predominantly applied in wheelchair-dependent patients. Activity-focused interventions of transfer, transition, sitting were trained most intensively in outcome group G1, while walking and swimming were increasingly trained in patients with moderate and good walking outcomes. Physical therapy interventions of assistive and active trainings as well as corresponding training environments changed with the recovery of locomotor function.

Conclusions Physical therapy of locomotor function is targeted to individual patients' conditions and becomes adjusted to the progress of ambulation. Although the involved clinical sites were not following explicitly standardized rehabilitation programs, common patterns can be discerned which may form the basis of prospective standardized programs.

Martina Franz and Lea Richner contributed equally to this work.

Electronic supplementary material The online version of this article (https://doi.org/10.1038/s41393-017-0007-5) contains supplementary material, which is available to authorized users.

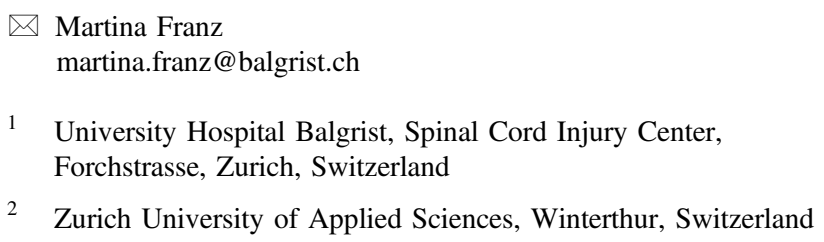

2 Zurich University of Applied Sciences, Winterthur, Switzerland

\section{Introduction}

Spinal cord injury (SCI) affects patients' independence and quality of life dramatically [1,2]. Novel neuroprotective and neuroregenerative treatment interventions should improve functional recovery beyond compensation and

3 Spinal Cord Injury Center, Heidelberg University Hospital, Heidelberg, Germany

4 European Multi-Center Study in Spinal Cord Injury (EMSCI), Zurich, Switzerland

5 BG Hospital, Center for Spinal Cord Injuries, Murnau am Staffelsee, Germany 
Table 1 Patient and injury characteristics

\begin{tabular}{lllll}
\hline Outcome groups & $\begin{array}{l}\text { Overall } \\
n=80\end{array}$ & $\begin{array}{l}\text { G1 } \\
n=41\end{array}$ & $\begin{array}{l}\text { G2 } \\
n=19\end{array}$ & $\begin{array}{l}\text { G3 } \\
n=16\end{array}$ \\
\hline Male/ female [n] & $59 / 21$ & $29 / 12$ & $15 / 4$ & $13 / 3$ \\
Age at injury, median (IQR), [years] & $50.0(35.5)$ & $55.0(30.0)$ & $51.0(36.8)$ & $31.5(23.5)$ \\
Cause of SCI & & & \\
$\quad$ Ischemic/ traumatic/ missing [n] & $6 / 73 / 1$ & $6 / 35 / 0$ & $0 / 19 / 0$ & $0 / 16 / 0$ \\
$\quad$ LOS, median (IQR), [days] & $99.0(57.0)$ & $111.0(51.0)$ & $87.0(61.8)$ & $72.5(40.0)$ \\
NLI and AIS at acute I & & & & \\
$\quad$ C1-C4, AIS B/C/D [n] & $1 / 5 / 14$ & $1 / 5 / 5$ & $0 / 0 / 5$ & $0 / 0 / 4$ \\
C5-C8, AIS B/C/D [n] & $3 / 3 / 10$ & $3 / 2 / 2$ & $0 / 1 / 3$ & $0 / 0 / 4$ \\
T1-S5, AIS B/C/D [n] & $8 / 6 / 10$ & $4 / 5 / 3$ & $4 / 1 / 2$ & $0 / 0 / 4$ \\
Missing [n] & 20 & 11 & 3 & 4 \\
\hline
\end{tabular}

spontaneous recovery [3, 4]. Clinical trials investigating such novel therapeutic strategies in acute/subacute SCI frequently state that rehabilitative therapies applied during such trials represent so called "conventional therapies" and are rather indistinguishable between treatment groups and participating clinical sites. However, "conventional therapy" is not formally defined or specified and may vary significantly between clinical sites treating spinal cord injured patients. In addition, common detailed rehabilitation programs are not systematically implemented [5], although principle guidelines for rehabilitation exist [6]. Instead, therapists are left to treat each patient to their best knowledge, potentially leading to significant variation in volume, intensity and type of training that patients undergo during rehabilitation.

Previous studies have been aimed at gaining insight in the amount and content of physical therapy interventions [7-9]. Currently, it is acknowledged that there is considerable variation in the content and duration of rehabilitation programs and their physical therapies [7, 10]. However, there are only few studies that describe the underlying patterns of physical therapy interventions, i.e., which kind of patients are provided with what kind of therapy when over the course of rehabilitation and what the effectiveness of such therapeutic interventions was [7]. In order to answer these questions, the content and duration of therapeutic interventions need to be documented with greater level of detail while simultaneously recording the extent of locomotor function of the patient.

Methods for documenting physical therapy interventions need to be practicable in day-to-day rehabilitative activities (preferably integrated into the routine work process) and eventually in clinical trials. One approach for the documentation of physical therapy after SCI was proposed in the SCIRehab Project [11]. Taxonomies were developed for all therapy disciplines participating in the rehabilitation process and focused on the relationship between therapy, patient characteristics, and outcome [11]. Another study conducted in Europe and Australia followed a similar approach [12] and invented a system for the SCI rehabilitation to code the content of physical, sport and occupational therapy; the Spinal Cord Injury - Intervention Classification System (SCI-ICS) [12]. It is a reliable [13] and feasible [14] system focused on the comparison of therapy content between different centers [7]. However, neither of the above-mentioned studies documented in parallel the neurological and functional condition of the patients over time. The walking ability of patients with incomplete paraplegia has a large potential for recovery during the weeks following the trauma [15], though it remains unclear whether the physical therapy content and dosage responds appropriately to these changes. The aim of this study (termed Mapping of Locomotor Training (MALT)) was to evaluate the content, timing and duration of physical therapy during in-patient rehabilitation in relation to changes of locomotor function within the European Multicenter Study on Spinal Cord Injury (EMSCI) network.

\section{Methods}

\section{Study design and inclusion criteria}

The MALT project is an observational multicenter study embedded in the EMSCI network (http://www.emsci.org). Six participating EMSCI network centers contributed to this study (University Hospital Balgrist, Spinal Cord Injury Center Zürich, Switzerland; Heidelberg University Hospital, Spinal Cord Injury Center, Germany; Trauma Center Murnau, Germany; Clinic for Orthopedics Hessisch-Lichtenau, Germany; Hospital Nacional de Parapléjicos, Toledo, Spain; Institut Guttman, Barcelona, Spain). Patients hospitalized for primary rehabilitation due to acute traumatic or ischemic incomplete SCI were enrolled. The study included lesion severity of American Spinal Injury Association Impairment Scale (AIS) grade B to D according to the 
International Standards of Neurological Classification of SCI [16] as well as all neurological levels of injury (NLI). Enrolled patients provided written consent to participate in the EMSCI project and this study.

\section{Data collection}

Registered physical and sports therapists recorded all administered physical and sports therapies without influencing or changing the interventions of these sessions. The therapy frequency was not fixed (i.e., therapists and patients were free to schedule therapy sessions) but the average frequency of physical therapy sessions was $2.1(0.7)$ per therapy day. Eligible patients were enrolled consecutively into the study except for periods, during which shortage of staff prohibited proper daily recording of physical therapy interventions. Data recording was performed between November 2013 and August 2016 from retrieval of informed consent until discharge from the rehabilitation center. Each participating center collected data in accordance with national ethics regulations (EMSCI project nr. EK-03/2004 (PB-2016-00293)). General information of patient demographics (age at injury, gender), injury characteristics (date of injury (DOI), cause of trauma) and length of stay (LOS) at the rehabilitation center was extracted from the EMSCI database. In addition, data about the neurological level of injury (NLI) and AIS grade were used from examination stage acute I (16-40 days after DOI), in accordance with the EMSCI study protocol (Table 1). The therapy interventions were logged using the SCI-ICS classification system [12], which uses 107 five-digit codes that are grouped into three main levels (body function and structure, basic activities, complex activities) and complemented by codes for equipment and other categories (SITable 1). Documentation sheets (SI-Table 2), available in German and English, were distributed to the participating centers and allowed therapists to record (1) general session information (e.g., session date, profession of therapist, individual or group session type), (2) the functional status of the patient using the three scoring items of the Spinal Cord Independence Measurement (SCIM) III [17] (item 12 for Mobility indoors $(-10 \mathrm{~m})$, item 13 for Mobility on moderate distances $10-100 \mathrm{~m}$, and item 15 for stair management), and (3) specific codes for the performed interventions including the intervention duration and whether and why therapy was canceled, affected, or shortened.

\section{Instruction of study protocol}

Leading therapists of the six participating centers were trained using SCI-ICS workshops and manuals [12]. Training at the workshop comprised an intensive theoretical SCI-ICS training and practical demonstrations of the coding of video recorded therapy sessions. After workshop completion, trained therapists instructed registered physical and sports therapists of their teams to record each therapy. Questions and concerns arising during data collection were continuously discussed and evaluated with the coordinating center. Collected data in the form of documentation sheets were transcribed from paper into an electronic database and centrally archived at the Research Department of the Spinal Cord Injury Center, University Hospital Balgrist, Zurich, Switzerland. Data sets with a recording period of less than 15 therapy days or EMSCI drop out patients were excluded from further analysis. Random samples were double checked for typing errors and a threshold of 5\% of errors was set (5 of 80 entered records yielded a typing error quotum of $3.75 \%)$.

\section{Definition of outcome groups}

To compare therapy across patients showing different trajectories of recovery, three discrete outcome groups were defined based on recovery of locomotor function. The grouping was based on the improvement of patients mobility on moderate distances $(10-100 \mathrm{~m}$; SCIM III mobility sub score, item 13) within the first and last ten recorded therapy days, which was independent from the NLI and AIS grading. Outcome group G1 included patients who were using a wheelchair (SCIM rating: 1-2) or required total assistance (SCIM rating: 0). Outcome group G2 consisted of patients who regained moderate walking capacity (SCIM rating: $3-5$ ) and patients in outcome group G3 entailed patients that regained good walking capacity (SCIM rating: 6-8).

\section{Transformation of the SCI-ICS into domains of rehabilitation}

To appreciate differences in therapy content, all SCI-ICS codes were classified on four layers in accordance with the International Classification of Functioning disability and Health (ICF) framework [18] (SI-Table 3). The first and second layers define the level of the intervention; body function/-structure, activity or everyday activity, and passive, assistive or active, respectively. The third layer defines the body function/-structure to be modulated by the intervention (e.g., tone, joint) or the activity or everyday activity to be trained by the intervention (e.g., walking, sitting). The fourth layer contains information about the intervention sites (e.g., water, treadmill) or the applied device (e.g., driven gait orthosis (DGO), electrical-wheelchair, electro-stimulation).

\section{Statistical analysis}

To compensate for different LOS of patients and the variable amount of received therapy, the first, middle and last 


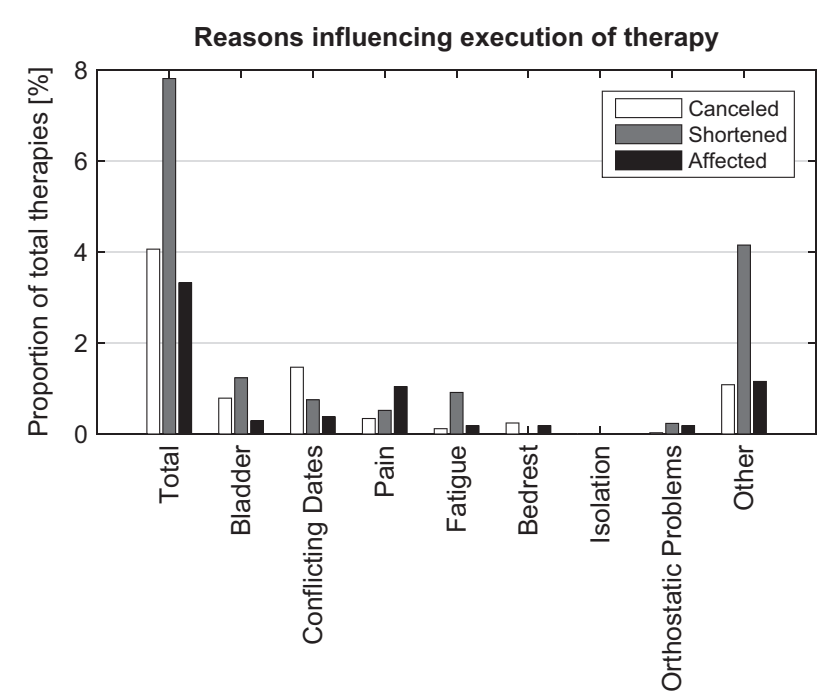

Fig. 1 Overview of the proportion of incomplete therapy sessions with the corresponding reasons

five therapy days were compared and percentages of specific therapy content calculated in relation to total therapy time. The median of this percentage for each outcome group was compared. As distributions were non-normal, nonparametric tests were used for statistical analysis; the significance level was set $p<0.05$. Therapy content was compared between outcome groups using the Kruskal-Wallis-Test $(\mathrm{KW})$ for each item of layer three (e.g., strength, walking, washing oneself, SI-Table 3). Where eligible, pairwise Dunn-Sidak (DS) comparisons were performed. To test for significant differences over time within each outcome group, a Friedman-Test was used. Statistical analyses were performed using MATLAB 2015b. If not mentioned otherwise, results including numbers in figures and tables are presented as median and interquartile range (IQR) in brackets.

\section{Results}

\section{Sample size}

Patient, injury characteristics and the distribution of the NLI and AIS grading of the different outcome groups are provided in Table 1. Following the inclusion criteria, data sets of 80 out of 88 initially enrolled patients were further analyzed. Four patients were excluded because the inclusion criteria were no longer met, and four other patients were excluded because the required minimum of 15 recorded therapy days was not met. Patients in outcome group G3 (median age 31.5 (23.5) years) were significantly younger than those in G1 (median age 55.0 (30.0) years, KW $(n=$ 76): $p=0.01$, DS $p=0.01$ ) and G2 (median age 51.0 (36.8) years, DS $p=0.03)$. Recording of therapy treatments started approximately 6.0 (16.5) days following the admittance to the rehabilitation center (data not shown).

\section{Characteristics of physical and sports therapy sessions}

In total, 10,731 physical and sports therapy sessions were provided to above-mentioned 80 patients and an additional $454(4.2 \%)$ therapies were planned but canceled, mainly due to conflicting dates (data not shown). Of all the applied sessions, $1244(11.6 \%)$ physical and sports therapy sessions could not be completed as planned due to complete cancelation, shortening or interference with SCI related complications (e.g., bladder/ bowel incontinence, pain, fatigue, orthostatic problems, Fig. 1).

Patients arrived at the rehabilitation center in median 9.0 (9.5) days after DOI and the median overall LOS was 99.0 (57.0) days (Table 1). The total time of therapy spent per patient added up to median 4510.0 (3280.0) minutes distributed over median 109.0 (80.0) therapy sessions on 56.0 (38.0) days in median. The average frequency of physical therapy sessions per day was $2.1(0.7)$ in median, with each session lasting in median 45.0 (30) min. There was a significant difference in the amount of therapy sessions during the first $<$ last $<$ middle five days of recorded therapies (Friedman-test $p<0.01$, post-hoc: DS, first vs. middle: $p<$ 0.01 , first vs. last $p=0.01$, middle vs. last $p=0.01$ ). Moreover, there were significantly more group therapies during the middle and last five days compared to the first five days (Friedman-test, $p<0.01$, post-hoc: DS, $p<0.01$ for both). Of all therapies, 25.0 (27.4)\% were performed in groups, while the remainder consisted of individual therapy sessions. Strength training was the most commonly performed intervention with 28.2 (20.8)\% of all therapy minutes, followed by walking training with 15.1 (17.6)\% of all therapy minutes (Table 2).

\section{Changes in locomotor capacity and therapeutic interventions}

At the initial assessment, the majority of patients either required total assistance (37 patients, $46.3 \%$ ) or used a wheelchair for mobility over $10-100 \mathrm{~m}$ (39 patients, $48.8 \%$ ), while only four patients (4.9\%) already initially showed moderate to good locomotor capacities (Fig. 2). The latter group was not further considered in the outcome groups. Recovery of a moderate to good walking function was detected in $61.5 \%$ of the initial wheelchair user patients as well as in $29.7 \%$ of the patients that initially required total assistance for mobility (Fig. 2). The two patients who continued to require total assistance for mobility both suffered from an incomplete (AIS C) cervical lesion with neurological level of lesion C2 and C3, and were 76 and 66 
Table 2 Overview of significant interventions

\begin{tabular}{|c|c|c|c|c|c|c|c|c|c|c|c|}
\hline & & \multirow{2}{*}{$\begin{array}{l}\text { Kruskal-Wallis }^{\mathrm{a}} \\
\text { Overall }\end{array}$} & \multicolumn{6}{|c|}{ Median (IQR) [\%] } & \multirow{2}{*}{$\begin{array}{l}\% \text { Of } \\
\text { patients } \\
(n=80)\end{array}$} & \multirow{2}{*}{$\begin{array}{l}\text { Total } \\
\text { hours } \\
(h)\end{array}$} & \multirow{2}{*}{$\begin{array}{l}\text { Median per } \\
\text { week } \\
(\mathrm{min})\end{array}$} \\
\hline & & & G1 & $\begin{array}{l}p \text {-values } \\
\text { G1 vs. G2 }\end{array}$ & $\mathrm{G} 2$ & $\begin{array}{l}p \text {-values } \\
\text { G1 vs. G3 }\end{array}$ & G3 & $\begin{array}{l}p \text {-values } \\
\text { G2 vs. G3 }\end{array}$ & & & \\
\hline \multirow[t]{9}{*}{$\begin{array}{l}\text { Body function/ } \\
\text { - structure }\end{array}$} & Strength & $<0.05^{\mathrm{a}}$ & $\begin{array}{l}23.8 \\
(24.6)\end{array}$ & 0.27 & $\begin{array}{l}31.2 \\
(18.0)\end{array}$ & 0.07 & $\begin{array}{l}35.2 \\
(19.5)\end{array}$ & 0.89 & 100.0 & 2175.6 & 94.3 \\
\hline & Stretching & 0.28 & $\begin{array}{l}2.1 \\
(3.7)\end{array}$ & & $\begin{array}{l}2.4 \\
(4.5)\end{array}$ & & $\begin{array}{l}1.0 \\
(2.4)\end{array}$ & & 85.0 & 270.0 & 6.7 \\
\hline & Tone & $<0.01^{\mathrm{a}}$ & $\begin{array}{l}3.7^{\mathrm{b}, \mathrm{c}} \\
(3.8)\end{array}$ & $0.02^{\mathrm{b}}$ & $\begin{array}{l}1.7^{\mathrm{b}} \\
(1.1)\end{array}$ & $0.01^{\mathrm{c}}$ & $\begin{array}{l}0.8^{\mathrm{c}} \\
(3.0)\end{array}$ & 0.96 & 81.3 & 271.2 & 8.2 \\
\hline & Joint & $0.02^{\mathrm{a}}$ & $\begin{array}{l}2 \\
(4.6)\end{array}$ & 0.05 & $\begin{array}{l}0.1 \\
(4.1)\end{array}$ & 0.07 & $\begin{array}{l}0.3 \\
(1.6)\end{array}$ & 1.00 & 71.3 & 211.0 & 3.5 \\
\hline & Sensibility & 0.99 & $\begin{array}{l}0.0 \\
(0.6)\end{array}$ & & $\begin{array}{l}0.0 \\
(0.6)\end{array}$ & & $\begin{array}{l}0.0 \\
(0.5)\end{array}$ & & 40.0 & 39.0 & 0.0 \\
\hline & Pain & 0.84 & $\begin{array}{l}0.5 \\
(2.5)\end{array}$ & & $\begin{array}{l}1.2 \\
(1.8)\end{array}$ & & $\begin{array}{l}0.8 \\
(2.3)\end{array}$ & & 62.5 & 89.0 & 1.7 \\
\hline & Skin & 0.98 & $\begin{array}{l}0.0 \\
(0)\end{array}$ & & $\begin{array}{l}0.0 \\
(0)\end{array}$ & & $\begin{array}{l}0.0 \\
(0)\end{array}$ & & 18.8 & 14.0 & 0.0 \\
\hline & Cardio & 0.25 & $\begin{array}{l}0.0 \\
(0.5)\end{array}$ & & $\begin{array}{l}0.0 \\
(1.2)\end{array}$ & & $\begin{array}{l}0.6 \\
(2.2)\end{array}$ & & 41.3 & 230.0 & 0.0 \\
\hline & Respiratory & $<0.01^{\mathrm{a}}$ & $\begin{array}{l}0.4^{\mathrm{b}, \mathrm{c}} \\
(2.6)\end{array}$ & $0.02^{\mathrm{b}}$ & $\begin{array}{l}0.0^{\mathrm{b}} \\
(0.3)\end{array}$ & $0.04^{\mathrm{c}}$ & $\begin{array}{l}0.0^{\mathrm{c}} \\
(0.5)\end{array}$ & 1.00 & 46.3 & 85.0 & 0.0 \\
\hline \multirow[t]{9}{*}{ Activity } & Transfer & $<0.01^{\mathrm{a}}$ & $\begin{array}{l}4.3^{\mathrm{b}, \mathrm{c}} \\
(6.7)\end{array}$ & $<0.01^{\mathrm{b}}$ & $\begin{array}{l}1.1^{\mathrm{b}} \\
(1.3)\end{array}$ & $<0.01^{\mathrm{c}}$ & $\begin{array}{l}0.8^{\mathrm{c}} \\
(1.4)\end{array}$ & 0.79 & 83.8 & 261.0 & 7.6 \\
\hline & Transition & $<0.01^{\mathrm{a}}$ & $\begin{array}{l}9.1^{\mathrm{b}, \mathrm{c}} \\
(9.2)\end{array}$ & $<0.01^{\mathrm{b}}$ & $\begin{array}{l}2.8^{\mathrm{b}} \\
(3.5)\end{array}$ & $0.02^{\mathrm{c}}$ & $\begin{array}{l}2.3^{\mathrm{c}} \\
(5.0)\end{array}$ & 0.84 & 87.5 & 426.0 & 19.3 \\
\hline & Sitting & $<0.01^{\mathrm{a}}$ & $\begin{array}{l}6.7^{\mathrm{c}} \\
(14.1)\end{array}$ & 0.06 & $\begin{array}{l}2.7 \\
(5.8)\end{array}$ & $0.05^{\mathrm{c}}$ & $\begin{array}{l}1.4^{\mathrm{c}} \\
(9.8)\end{array}$ & 1.00 & 86.3 & 617.0 & 14.4 \\
\hline & Wheelchair & 0.26 & $\begin{array}{l}5.4 \\
(12.2)\end{array}$ & & $\begin{array}{l}6.5 \\
(9.0)\end{array}$ & & $\begin{array}{l}3.1 \\
(6.3)\end{array}$ & & 88.8 & 596.0 & 16.5 \\
\hline & Standing & 0.19 & $\begin{array}{l}6.3 \\
(6.3)\end{array}$ & & $\begin{array}{l}8.4 \\
(4.3)\end{array}$ & & $\begin{array}{l}7.4 \\
(5.9)\end{array}$ & & 93.8 & 531.0 & 26.0 \\
\hline & Walking & $<0.01^{\mathrm{a}}$ & $\begin{array}{l}9.8^{\mathrm{b}, \mathrm{c}} \\
(16.6)\end{array}$ & $<0.01^{\mathrm{b}}$ & $\begin{array}{l}24.5^{\mathrm{b}} \\
(21.1)\end{array}$ & $0.01^{\mathrm{c}}$ & $\begin{array}{l}18.0^{c} \\
(18.3)\end{array}$ & 0.97 & 87.5 & 1158.8 & 52.7 \\
\hline & Swimming & $0.02^{\mathrm{a}}$ & $\begin{array}{l}0.0^{\mathrm{c}} \\
(0)\end{array}$ & 0.77 & $\begin{array}{l}0.0 \\
(0.6)\end{array}$ & $0.01^{\mathrm{c}}$ & $\begin{array}{l}0.2^{\mathrm{c}} \\
(4.3)\end{array}$ & 0.22 & 25.0 & 59.0 & 0.0 \\
\hline & & & & & \multicolumn{4}{|c|}{ TOTAL of 16 interventions above } & & 7039 & $\begin{array}{l}360 \\
(189)\end{array}$ \\
\hline & & & & & TOTA & of all inter & rentions & & & 7385 & $\begin{array}{l}381 \\
(204)\end{array}$ \\
\hline
\end{tabular}

${ }^{\text {a Significant difference if } p<0.05}$

${ }^{\mathrm{b}}$ Significant difference between G1 and G2 (post-hoc with Dunn-Sidac)

${ }^{\mathrm{c}}$ Significant difference between G1 and G3 (post-hoc with Dunn-Sidac)

years of age. There was no significant difference between outcome groups regarding the delay between DOI and the admission to rehabilitation (KW $(n=76): p=0.11)$, the number of therapy sessions (KW $(n=76): p=0.26)$ nor total therapy time (KW $(n=76): p=0.46)$. Detailed results of the applied statistical tests are shown in Table 2. However, outcome group G1 (111.0 (51) LOS) had a significantly longer in-patient rehabilitation than outcome group G3 (KW $(n=76): p=0.01$, DS: $p<0.01) 72.5$ (40) LOS. The proportion of group therapy vs. individual 
therapy was not significantly different between all outcome groups (KW $(n=76): p=0.20)$.

The temporal change in administered physical and sports therapeutic interventions during rehabilitation is illustrated in Fig. 3. Patients of G1 spent significantly greater portions of their physical therapy time focusing on tone, respiratory, transfer, transition and sitting while they spent less therapy time on walking than patients of G2 or G3. There were also specific differences in when specific activity or body function/-structure interventions were trained (Fig. 3, Table 2). For instance, during the first five days, therapy was significantly more often assistive than during the last five days (Friedman, $p=0.01$, DS, $p=0.01$ ) and significantly less active than during the last and middle five days (Friedman, $p=0.01$, Timepoint 1 vs. Timepoint 2: DS $p=0.04$, Timepoint 1 vs. Timepoint 3: DS $p=0.01$ ). There was no significant difference in the proportion of passive therapy (Friedman, $p=0.42$ ).

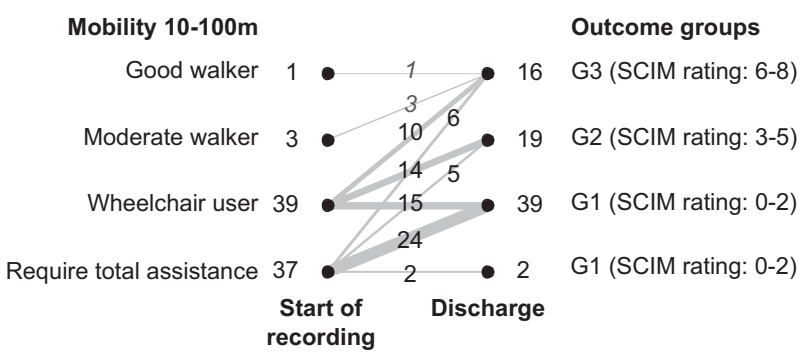

Fig. 2 Recovery of locomotor function. All patients except two participants without standing/walking capability showed a recovery of locomotor function. The four patients who initially demonstrated good or moderate walking function were excluded from analysis

\section{Changes in specific interventions}

The presented data illustrates that interventions change over time, which may be related to locomotor functionality (Figs. 4 and 5). Strength training (distinguished into "assisted by therapist, against gravity and against resistance") showed specific patterns of application across all outcome groups. While outcome group G1 continuously received all three categories of strength training throughout the time of rehabilitation, the training shifted towards more active strength training against resistance with increasing locomotor outcomes (Fig. 4). A similar distinction between outcome groups was in walking and ambulation therapy: the three outcome groups clearly had different patterns of applied interventions, e.g., trained different modalities at different stages within the rehabilitation (Fig. 5). DGO and treadmill trainings were applied throughout the rehabilitation process, while the latter interventions were reduced in patients with regained locomotor function in favor of more active and challenging training environments.

\section{Discussion}

This study performed on SCI patients undergoing primary in-patient rehabilitation within the EMSCI network, recorded all physical and sports therapy sessions for 80 patients with acute incomplete SCI. The involved SCI centers did not follow an explicitly standardized rehabilitation program and differences in national healthcare systems between the investigated countries and between the participating hospitals needs to be investigated in dedicated larger studies. For this study, the data from the investigated centers were treated as a single cohort to discern major patterns in the
Fig. 3 Heat map illustrating the intervention intensity. Each box corresponds to an outcome group and represents $20 \%$ of the recorded physical and sports therapies from the start of documentation to patient discharge. The color of the boxes represents the median proportion of an intervention compared to the total of all recorded interventions. The darker the color, the more intensively the intervention is trained (see legend)

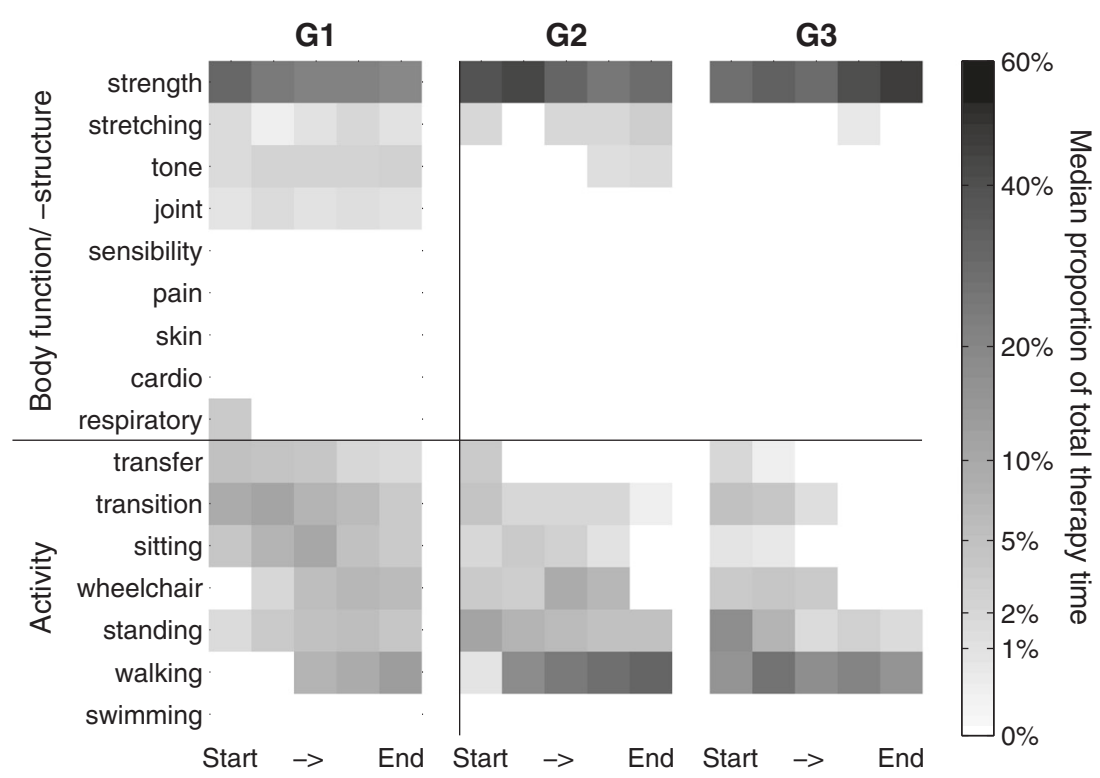


Fig. 4 Details of strength training content. This figure shows the change in the content of strength training (divided into three degrees of difficulty: assisted by therapist, against gravity and against resistance) of the three outcome groups (G1-G3) over the course of rehabilitation. The box plots consist of the median (thick white line), the $25 \%$ - and $75 \%$ percentile ranges, the $1.5 * \mathrm{IQR}$ (whiskers) and outliers (3*IQR, black dots)

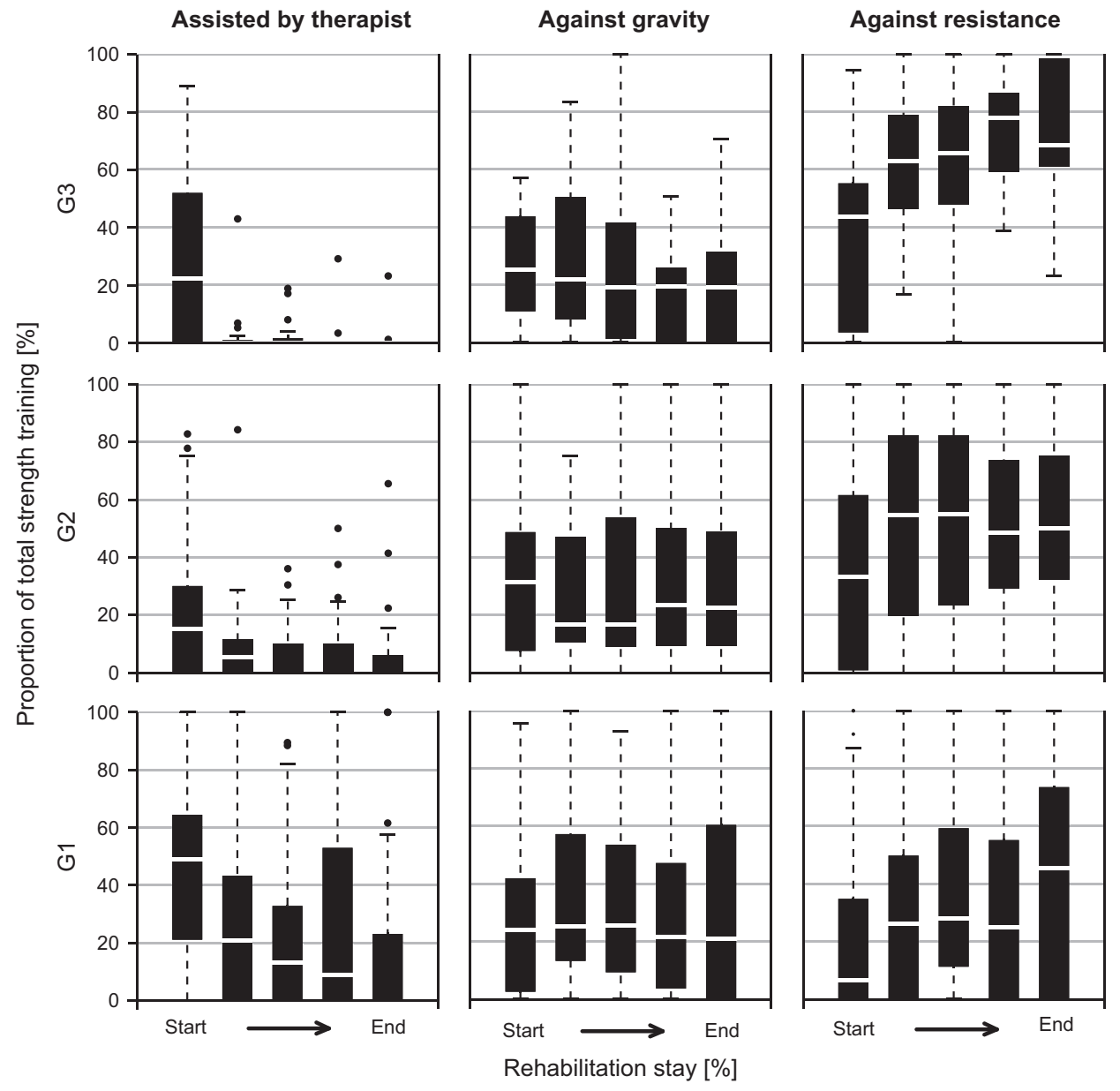

therapy content and duration as applied in the participating centers.

Overall, it seems that training interventions were targeted according to the level of impairment and adjusted over time responding to the recovery of locomotor function, although at this point it cannot be determined whether the rehabilitative interventions followed the improving locomotor function or whether improvement of locomotor function was promoted by the quality/quantity of rehabilitative interventions. Previous studies have focused on therapy content of rehabilitation following acute SCI but did not record changes of the mobility status in these patients. One previous cross-sectional study [19], although limited to four weeks, documented physical, occupational and sports therapy sessions with a focus on therapy duration. It reported approximately equal therapy proportions spent on the level of body function/-structure and on the level of activity [19]. Similar findings were reported in a study from Australia and Norway [7], but likewise, these studies did not simultaneously record functional recovery.

The length of in-patient rehabilitation observed in this study (median 99.0 (57.0) days) was considerably longer than in the SCIRehab study in the US (approximate mean of
$55.7 \mathrm{~d}$ (SD 36.6)) [8], while the delay between injury and admittance to rehabilitation was shorter in the European study (in mean 9.0 (9.5) days) compared to the SCIRehab study (approximately 32 days) [10]. However, both these differences do not relate to the therapy content or rehabilitation goals, and are likely confounded by organizational structures in the national healthcare systems in the respective countries.

Concerning the duration of therapy sessions, a literature comparison shows that previously observed therapy mean session durations of $38.9 \mathrm{~min}$ (SD 8.0) in Norway and 43.3 min (SD 6.3) in Australia [7] are comparable to the median duration of therapy sessions observed in this study (45.0 (30.0) $\mathrm{min}$ ) while in the Netherlands, the average therapy session duration was lower (28.1 min (SD 7.7) [7]. Furthermore, the average time of physical therapy per week of about 442.3 (SD 127.2) $\min (n=132)$ in the latter study was rather comparable to the median 381.1 (203.7) $\mathrm{min}$ in this study. The same is true for the proportion between single and group therapy, with $23 \%$ in the SCIRehab study [10] compared to $25 \%$ in this study.

Thus, although the set-up of rehabilitation programs may show differences between countries (as discussed above), 

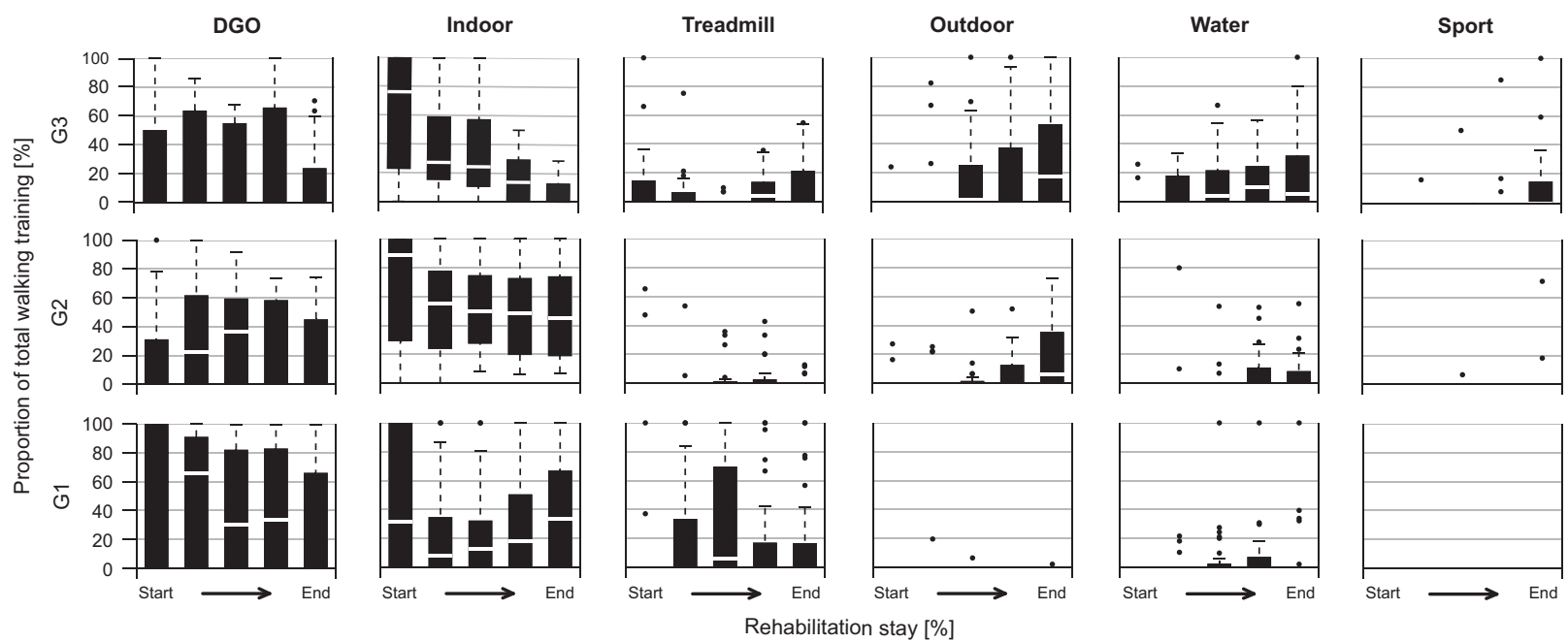

Fig. 5 Details of walking training content. This figure shows the change in the content of walking training (divided into the six different therapy types DGO, Indoor, Treadmill, Outdoor, Water und Sport) of the three outcome groups (G1-G3) over the course of rehabilitation.

The box plots consist of the median (thick white line), the $25 \%$ - and $75 \%$ - percentile ranges, the $1.5 * \mathrm{IQR}$ (whiskers) and outliers $(3 * \mathrm{IQR}$, black dots)

metrics related to therapy content and time allocations appear to be rather comparable between countries. Therefore, our findings in a reasonable amount of patients and $>10.500$ investigated sessions of therapy across six centers may represent gross common standards in specialized European SCI centers. However, this still needs to be confirmed in larger multinational studies.

\section{Training of locomotor activity}

Our results show that most body function/-structure trainings were applied rather equally across the different outcome groups (stretching, sensibility, pain; Table 2), whereas the training of tone, and respiration differed between the outcome groups. In contrast, the training of activities was rather related to the extent of functional impairment, with the time and content of trainings changing with the improvement of locomotor function (e.g., transfer, walking; Fig. 5, Table 2). These findings suggest that rehabilitation programs of locomotor function are generally composed of basic training elements (i.e., independent from the actual impairment of body functions) and by specific training elements that are adjusted to the extent of functional impairment (i.e., activities). This is in agreement with common practice in physical therapy for neurological disorders, where the execution of any activity training (i.e., joint movements by voluntary coordinated muscle activations) requires appropriate conditions of muscle tone, range of joint movements and afferent feedback (i.e., sensor function) to enable effective motor training. These requirements are relatively similar for motor tasks like reaching, standing and walking activities, where training benefits from highly task specific trainings are proportionally dependent on level of capacity [20, 21].

However, it is interesting to observe from the presented data that both treadmill and DGO training categories were applied for locomotor training with no obvious distinct relation to the actual functional condition: both were applied over a broad spectrum of locomotor capacities (Fig. 5). This has been observed previously [22], and may be related to the fact that such assisted training devices may serve broader locomotor capacities, i.e., may not only be applied in patients with complete but also different levels of incomplete SCI. In addition, structural (shortage of staff) and administrative (in some institutions, levels of reimbursement are partially influenced by the applied technological devices) constraints may affect the variable application of treadmill and DGO training in SCI rehabilitation.

\section{Strength training}

Our data shows that strength training represents the largest proportion of the overall training programs, which agrees with findings of other SCI studies [7, 10, 19]. Strength training is applied to a similar extent across the different outcome groups with a tendency toward proportionally more training in less impaired patients and to an increasing extent toward the end of rehabilitation (Fig. 4, Table 2). This finding aligns with reduced strength being one of the major challenges for SCI patients and one of the most intensively trained capacities $[5,10,19]$. This is due to the SCI itself, to secondary injuries (particularly of the upper and lower limbs) as well as disuse as SCI patients spend different amounts of time in acute care hospitals and are 
variably immobilized due to surgical or conservative treatment [5]. This in turn may lead to an additional decrease in muscle strength [19], which has to be retrained in subsequent rehabilitation. In addition, the adaptation and learning of compensatory movements require patients to train specific muscles [23] (such as upper limb muscles to perform wheelchair transfers) to develop the capacity to reliably execute demanding movements. The therapy content appears to adjust in response to the changes in the level of patients' impairments, which is illustrated by the tendency toward more active training and more skilled locomotor levels over time, corresponding to functional recovery. Over the course of rehabilitation, it is generally believed that a clinical condition improves (at least functionally and to some extent neurologically) with a simultaneously reduced dependency on physical assistance for mobility (ideally improving from sitting to standing and walking) [24]. Accordingly, physical therapy is targeted toward highest degrees of independent activities of everyday life [24]. This is also reflected by our results, which show that the applied training modalities and training environment become more challenging toward the end of inpatient rehabilitation (Figs. 3 and 5). Although this may seem intuitive, it has previously never been illustrated at this level of detail in a prospective multi-center study.

\section{Conclusions}

Although there are no standardized and detailed protocols of physical therapy in acute SCI beyond rather general (inter)national guidelines, this study shows that content and intensity of physical and sports therapy are implicitly linked to the profile of locomotor recovery throughout rehabilitation. Our results indicate that physical and sports therapy regimes are calibrated to patients' conditions and may adapt over time in response to the improvement of walking function. However, as therapy is not a one-size-fits-all product, physical and sports therapies need to be individually shaped according to locomotor function and its change over time. The provided matrix of data analysis can be applied to describe and assess rehabilitation programs. A standardized matrix may be applicable to improve rehabilitation programs and thereby contribute to further insights in the contribution of physical therapy for functional recovery.

\section{Data archiving}

All relevant data are within this manuscript and raw data are archived by the authors.
Acknowledgements We acknowledge all participating therapists of the Spinal Cord Injury Centers in Zurich, Heidelberg, Murnau, Hessisch-Lichtenau, Toledo, and Barcelona. This study was supported by the Wings for Life Foundation, the European Multicenter Spinal Cord Injury (EMSCI) network and the Clinical Research Priority Program (CRPP) from Neuro-Rehab. We thank Chris Easthope Schmidt for proofreading.

\section{Compliance with ethical standards}

Conflict of interest The authors declare that they have no competing interests.

\section{References}

1. Leduc BE, Lepage Y. Health-related quality of life after spinal cord injury. Disabil Rehabil. 2002;24:196-202.

2. Dijkers M. Quality of life after spinal cord injury: a meta analysis of the effects of disablement components. Spinal Cord. 1997; 35:829-40.

3. Curt A, Van Hedel HJ, Klaus D, Dietz V, EM-SCI Study Group. Recovery from a spinal cord injury: significance of compensation, neural plasticity, and repair. J Neurotrauma. 2008;25:677-85.

4. Wang Y, Wu M, Gu L, Li X, He J, Zhou L, et al. Effective improvement of the neuroprotective activity after spinal cord injury by synergistic effect of glucocorticoid with biodegradable amphipathic nanomicelles. Drug Deliv. 2017;24:391-401.

5. Harvey LA. Physiotherapy rehabilitation for people with spinal cord injuries. J Physiother. 2016;62:4-11.

6. Tweedy SM, Beckman EA, Geraghty TJ, Theisen D, Perret C, Harvey LA, et al. Exercise and sports science Australia (ESSA) position statement on exercise and spinal cord injury. J Sci Med Sport. 2016;20:422-3.

7. van Langeveld SA, Post MW, van Asbeck FW, Gregory M, Halvorsen A, Rijken $\mathrm{H}$, et al. Comparing content of therapy for people with a spinal cord injury in postacute inpatient rehabilitation in Australia, Norway, and The Netherlands. Phys Ther. 2011;91:210-24.

8. Teeter L, Gassaway J, Taylor S, LaBarbera J, McDowell S, Backus D, et al. Relationship of physical therapy inpatient rehabilitation interventions and patient characteristics to outcomes following spinal cord injury: the SCIRehab project. J Spinal Cord Med. 2012;35:503-26.

9. Whiteneck G, Gassaway J, Dijkers M, Backus D, Charlifue S, Chen $\mathrm{D}$, et al. Inpatient treatment time across disciplines in spinal cord injury rehabilitation. J Spinal Cord Med. 2011; 34:133-48.

10. Taylor-Schroeder S, LaBarbera J, McDowell S, Zanca JM, Natale A, Mumma S, et al. Physical therapy treatment time during inpatient spinal cord injury rehabilitation. J Spinal Cord Med. 2011;34:149-61.

11. Whiteneck G, Gassaway J, Dijkers M, Jha A. New approach to study the contents and outcomes of spinal cord injury rehabilitation: the SCIRehab project. J Spinal Cord Med. 2009; 32:251-9.

12. van Langeveld SA, Post MW, van Asbeck FW, Postma K, Ten Dam D, Pons K. Development of a classification of physical, occupational, and sports therapy interventions to document mobility and self-care in spinal cord injury rehabilitation. J Neurol Phys Ther. 2008;32:2-7.

13. van Langeveld SA, Post MW, van Asbeck FW, Ter Horst P, Leenders J, Postma K, et al. Reliability of a new classification system for mobility and self-care in spinal cord injury 
rehabilitation: the spinal cord injury-interventions classification system. Arch Phys Med Rehabil. 2009;90:1229-36.

14. van Langeveld SA, Post MW, van Asbeck FW, Postma K, Leenders J, Pons K. Feasibility of a classification system for physical therapy, occupational therapy, and sports therapy interventions for mobility and self-care in spinal cord injury rehabilitation. Arch Phys Med Rehabil. 2008;89:1454-9.

15. Burns SP, Golding DG, Rolle WA Jr, Graziani V, Ditunno JF Jr. Recovery of ambulation in motor-incomplete tetraplegia. Arch Phys Med Rehabil. 1997;78:1169-72.

16. Kirshblum SC, Burns SP, Biering-Sorensen F, Donovan W, Graves DE, Jha A, et al. International standards for neurological classification of spinal cord injury (revised 2011). J Spinal Cord Med. 2011;34:535-46.

17. Itzkovich M, Gelernter I, Biering-Sorensen F, Weeks C, Laramee MT, Craven $\mathrm{BCl}$, et al. The spinal cord independence measure (SCIM) version III: reliability and validity in a multi-center international study. Disabil Rehabil. 2007;29:1926-33.

18. World Health Organization. International classification of functioning, disability and health: ICF2001: World Health Organization (Geneva, Switzerland, 2001). Available online at: http://a pps.who.int/classifications/icfbrowser/. Accessed March 1st, 2017.
19. van Langeveld SA, Post MW, van Asbeck FW, ter Horst P, Leenders J, Postma K, et al. Contents of physical therapy, occupational therapy, and sports therapy sessions for patients with a spinal cord injury in three Dutch rehabilitation centres. Disabil Rehabil. 2011;33:412-22.

20. Galea MP. Physical modalities in the treatment of neurological dysfunction. Clin Neurol Neurosurg. 2012;114:483-88.

21. Girgis J, Merrett D, Kirkland S, Metz GA, Verge V, Fouad K. Reaching training in rats with spinal cord injury promotes plasticity and task specific recovery. Brain. 2007;130: 2993-3003.

22. Dobkin BH, Duncan PW. Should body weight-supported treadmill training and robotic-assistive steppers for locomotor training trot back to the starting gate? Neurorehabil Neural Repair. 2012;26:308-17.

23. Bye E, Harvey LA, Gambhir A, Kataria C, Glinsky JV, Bowden JL, et al. Strength training for partially paralysed muscles in people with recent spinal cord injury: a within-participant randomised controlled trial. Spinal Cord. 2016;55:460-5.

24. Harvey L. Management of spinal cord injuries: a guide for physiotherapists. Elsevier Health Sciences, 2008 (ed. Lisa Harvey, Published by Elsevier Health Sciences, Amsterdam, the Netherlands, 2008. ISBN: 9780443068584). 